\title{
Europäische Integration auf Unternehmensebene: die Schweizer Beteiligung an Europäischen Betriebsräten (EBR)
}

\author{
Patrick Ziltener* und Heinz Gabathuler* \\ *Institut für Soziologie, Universität Zürich, CH-8050 Zürich, ziltener@soziologie.uzh.ch, gabathuler@ \\ soziologie.uzh.ch.
}

\section{Abstracts \\ Europäische Integration auf Unternehmensebene: die Schweizer Beteiligung an Europäischen Betriebsräten (EBR)}

Der Beitrag untersucht das Ausmass und die Bestimmungsfaktoren der Beteiligung Schweizer Arbeitnehmer an Europäischen Betriebsräten (EBR) in transnationalen Konzernen. Die entsprechende EU-Richtlinie ist für Schweizer Arbeitnehmer nicht anwendbar; diese sind aber auf freiwilliger Basis in etlichen EBR vertreten. Unsere Resultate zeigen, dass über 150 EBR über Schweizer Vertreter verfügen, was ungefähr einem Drittel der Unternehmen mit EBR und Beschäftigten in der Schweiz entspricht. Unternehmen mit Hauptsitz in der Schweiz und solche mit vielen Beschäftigten in der Schweiz schliessen Schweizer Arbeitnehmer häufiger ein als andere. Zudem verfügen 50 Schweizer Unternehmen mit Niederlassungen in der EU über einen EBR.

Schlüsselwörter: Arbeitsbeziehungen, europäische Integration, Arbeitnehmermitwirkung, transnationale Konzerne, Europäische Betriebsräte

\section{Intégration européenne au niveau de l'entreprise: participation suisse aux comités d'entreprise européens (CEE)}

L'article examine les facteurs déterminants pour la participation des travailleurs suisses aux comités d'entreprise européens (CEE) dans des entreprises transnationales et considère l'ampleur de cette participation. La directive de l'UE à ce sujet n'est pas applicable aux travailleurs suisses; ceux-ci sont néanmoins représentés dans plusieurs CEE sur une base volontaire. Nos résultats indiquent que plus de 150 CEE disposent de représentants suisses, ce qui correspond à environ un tiers des entreprises ayant un CEE et des employés en Suisse. Les entreprises dont le siège principal est en Suisse et celles avec un grand nombre d'employés en Suisse incluent les travailleurs suisses plus souvent que les autres. En outre, 50 entreprises suisses avec des filiales dans l'UE disposent d'un CEE.

Mots-clés: Relations industrielles, intégration européenne, participation des travailleurs, entreprises transnationales, comités d'entreprise européens

\section{European Integration on Company Level: Swiss Participation in European Works Councils (EWC)}

The article examines the extent of and the determining factors for the participation of Swiss employees in European Works Councils (EWC) in transnational corporations. The respective EU directive is not applicable for Swiss employees; however they are represented on a voluntary basis in a number of EWCs. Our results show that more than 150 EWCs include Swiss representatives, which equals around one third of all companies with an EWC and employees in Switzerland. Companies headquartered in Switzerland as well as companies with a large number of employees in Switzerland are more likely to include the Swiss workforce than others. In addition, 50 Swiss companies with undertakings in the EU do have an EWC.

Keywords: Industrial relations, European integration, worker participation, transnational corporations, European Works Councils 


\title{
Europäische Integration auf Unternehmensebene: die Schweizer Beteiligung an Europäischen Betriebsräten (EBR)
}

\author{
Patrick Ziltener* und Heinz Gabathuler*
}

\section{Einführung und Fragestellung}

\subsection{Hintergrund: die EU-Richtlinie über Europäische Betriebsräte}

Dieser Aufsatz untersucht die Frage, in welchem Ausmass Europäische Betriebsräte (EBR) für die Arbeitnehmer in der Schweiz sowie für schweizerische Unternehmen von Bedeutung sind, obwohl die dieser Institution zugrunde liegende Richtlinie der Europäischen Union (EU) für die Schweiz rechtlich nicht anwendbar ist (Richtlinie 94/45/EG: Einsetzung eines Europäischen Betriebsrats oder die Schaffung eines Verfahrens zur Unterrichtung und Anhörung der Arbeitnehmer in gemeinschaftsweit operierenden Unternehmen und Unternehmensgruppen). Die Richtlinie geht auf das Projekt der damaligen Europäischen Kommission zurück, im Hinblick auf die Vollendung des Binnenmarktes dessen "soziale Dimension" zu erweitern (Ziltener 2000a). Ziel war, neben dem institutionalisierten Sozialdialog auf gesamtwirtschaftlicher und Branchenebene ein Instrument des Sozialdialogs auf Unternehmensebene zu schaffen. Konstituiert wurde damit das Recht der europäischen Arbeitnehmer auf Information und Konsultation in länderübergreifenden Angelegenheiten - also beispielsweise bei Restrukturierungen, welche Standorte in mehreren EU-Staaten betreffen, bei der beabsichtigten Verlagerung von Betrieben oder Betriebsteilen von einem EU-Staat in den anderen, sodann bei Übernahmen und Zusammenschlüssen. Die Richtlinie gilt für Unternehmen mit mindestens 1000 Beschäftigten in Staaten der EU und des Europäischen Wirtschaftsraumes (EWR), wovon in mindestens zwei Staaten je mindestens 150 Arbeitnehmer beschäftigt sein müssen. Unternehmen mit Hauptsitz ausserhalb von EU / EWR haben laut Richtlinie eine Niederlassung in der EU mit den Verhandlungen über die Einrichtung eines EBR zu beauftragen. Ein EBR ist komplementär zu den bestehenden Arbeitnehmervertretungen nach nationalem Recht; eine Mitbestimmung der Arbeitnehmer auf Unternehmensebene ist nicht vorgesehen. ${ }^{1}$ Die EBR-Richtlinie hat also keinerlei unternehmensrechtliche Relevanz, sondern ist auf der Ebene des Arbeitsrechts angesiedelt (Streeck 1997). Es handelt sich aber immerhin um die erste Institution des kollektiven Arbeitsrechts auf europäischer Ebene (Platzer und Müller 2011, 72), die zudem perspektivisch

\footnotetext{
* Institut für Soziologie, Universität Zürich, CH-8050 Zürich, ziltener@soziologie.uzh.ch, gabathuler@soziologie.uzh.ch. Unter Mitarbeit von André Walter, M. A., Universität St. Gallen. Zur Unterscheidung von Mitwirkung und Mitbestimmung vgl. Böhringer (2003).
} 
«in mehrfacher Hinsicht als dynamischster Pol der Europäisierung der Arbeitsbeziehungen" gesehen wird: Platzer $(2002,113,115)$ beobachtete «bei einer hohen Zahl von Europäischen Betriebsräten (...) eine bemerkenswerte Entwicklungsdynamik (...), hin zu einer neuen europäischen Ebene betrieblicher Arbeitsbeziehungen». Auch wenn spätere Forschungen zu ernüchternderen Ergebnissen in Bezug auf den «europäischen» Charakter der Institution EBR gelangen (Kotthoff 2006; Whittall et al. 2007; Waddington 2011), bleibt doch die Frage, ob und inwieweit Schweizer Arbeitnehmer davon betroffen sind.

Bis zu ihrem Inkrafttreten im September 1996 konnten gemäss Art. 13 der Richtlinie Vereinbarungen mit nicht näher definierten Vertretern der Arbeitnehmerseite abgeschlossen werden («Art. 13-Vereinbarungen»). Für Unternehmen, in welchen bis dahin noch keine Verhandlungen aufgenommen worden waren, sind Verhandlungen zwischen dem Management und in den verschiedenen Standortländern gewählten ArbeitnehmervertreternInnen sowie Mindeststandards für die $\mathrm{zu}$ regelnden Gegenstände vorgeschrieben («Art. 6-Vereinbarungen»). Kommt in einem solchen Verfahren keine Vereinbarung zustande, wird ein EBR gemäss den bezüglich Zusammensetzung und Rechten des Gremiums detaillierten subsidiären Bestimmungen der Richtlinie eingerichtet. Dies ist bisher nur in wenigen Fällen geschehen. Platzer $(2002,111)$ spricht zutreffend von «regulierter Selbstregulierung». Bei den Verhandlungen zur Errichtung von EBR in einigen Schweizer Unternehmen waren Funktionäre von Schweizer Gewerkschaften im Auftrag der jeweiligen europäischen Gewerkschaftsdachverbände federführend dabei; eine kleinere Anzahl von EBR wird auch heute noch aktiv von Schweizer Gewerkschaften betreut. ${ }^{2}$ Der Europäische Gewerkschaftsbund (EGB) bemühte sich in den 1990er-Jahren darum, in den Verhandlungen mit den Konzernleitungen über die Einrichtung von EBR eine Vertretung auch der Schweizer und der damals ebenfalls noch nicht obligatorisch einbezogenen britischen ${ }^{3}$ Standorte zu erwirken (Baumann 1997, 61; Waddington 2011, 40; Wyler 2012, 219). In der Schweiz wurde jüngst auf parlamentarischer Ebene ein Versuch unternommen, die EBR-Richtlinie für die Arbeitnehmer in der Schweiz verbindlich zu erklären. ${ }^{4}$

2009 wurde die Richtlinie neu gefasst (Richtlinie 2009/38/EG). In einigen Punkten wurden wie von den Gewerkschaften auf europäischer Ebene gewünscht

2 Die EBR von Rieter, Holcim, Forbo und Autoneum vom Unia-Zentralsekretariat, der EBR von Lonza vom Syna-Regionalsekretariat in Visp.

3 Das Vereinigte Königreich hatte darauf verzichtet, das Sozialpolitische Protokoll des Maastrichter Unionsvertrags von 1991 zu unterzeichnen. Nur so wurden Mehrheitsentscheidungen in Fragen des Arbeitsrechts (inklusive der Mitwirkung der Arbeitnehmer) und somit unter den gegebenen politischen Verhältnissen die Verabschiedung der EBR-Richtlinie ermöglicht (Ziltener 2000a). 1997 unterzeichnete die Regierung von Tony Blair das Sozialpolitische Protokoll, womit die Richtlinie für das Vereinigte Königreich verbindlich wurde.

42011 reichte Nationalrätin Susanne Leutenegger Oberholzer (Sozialdemokratische Partei, Baselland) eine Motion ein unter dem Titel «Mitwirkung in EU-Betriebsräten: Gleiche Rechte für Schweizer Arbeitnehmende». Sie wurde von der Mehrheit des Nationalrats auf Antrag des Bundesrats im Sommer 2012 verworfen. 
die Rechte und Kompetenzen der EBR erweitert, ohne allerdings den grundlegenden Charakter der Bestimmungen, namentlich die Beschränkung auf Information und Konsultation und inhärenten Voluntarismus in der Umsetzung zu verändern (Waddington 2011, 206-207; Thüsing 2011, 313-318).

Separat ist die Mitwirkung der Arbeitnehmer in Europäischen Aktiengesellschaften geregelt (Richtlinie 2001/86/EG: Ergänzung des Status der Europäischen Gesellschaft (Societas Euopaea, SE) hinsichtlich der Beteiligung der Arbeitnehmer). Auch hier gilt das Prinzip des Vorrangs freiwilliger Vereinbarungen; allerdings ist für nach bisherigem nationalem Recht bereits bestehende Formen der Mitbestimmung auf Unternehmensebene ein Bestandesschutz vorgesehen. Dies kann zur Folge haben, dass an Stelle bisheriger nationaler Arbeitnehmervertretungen (z. B. einem Gesamtoder Konzernbetriebsrat in Deutschland) der EBR bzw. SE-Betriebsrat die Vertretung der Arbeitnehmer in das höchste Aufsichtsgremium delegieren kann; diesem indirekt also Mitbestimmungs- und nicht nur Mitwirkungsbefugnisse zukommen.

\subsection{Fragestellung}

Zuerst wird untersucht, in welchem Ausmass die Arbeitnehmer der schweizerischen Standorte transnationaler Unternehmen in Europäischen Betriebsräten vertreten sind. Dies geschieht auf freiwilliger Basis, weil die Richtlinie ja für die Schweiz nicht anwendbar ist. Diese Frage ist von Bedeutung, weil es sich bei der Vertretung von Schweizer Arbeitnehmern in EBR nicht nur um eine Form der selektiven Integration des Nicht-EU-Landes Schweiz in die EU handelt, sondern anders als etwa im Bereich der Personenfreizügigkeit auf dem Arbeitsmarkt um eine «Europäisierung der Arbeitsbeziehungen" auf rein voluntaristischer Basis und ohne eigentliche Rechtsgrundlage, weder in der Schweiz noch in der EU. Sie ist zudem von Relevanz, als im Zuge von «Verbetrieblichungstendenzen» der Arbeitsbeziehungen, etwa im Bereich der Lohnverhandlungen in verschiedenen Zweigen der verarbeitenden Industrie in der Schweiz, den gewählten betrieblichen Arbeitnehmervertretungen eine verstärkte Bedeutung zukommt (Oesch 2011). Die Frage, ob EBR in diesem Kontext eine bedeutende Rolle spielen (Sadler 2000), muss hier offen bleiben. Ziel der Untersuchung ist hingegen, die Frage nach den Determinanten einer Schweizer Beteiligung zu beantworten: Welche Faktoren führen zu einem Einschluss Schweizer Arbeitnehmer, welche wirken in Richtung einer Nichtbeteiligung?

Als Weiteres wird die Frage nach der Umsetzung der EBR-Richtlinie bei transnationalen Unternehmen mit Hauptsitz in der Schweiz behandelt. Denn auch wenn die Teilnahme von Schweizer Arbeitnehmervertretern in EBR auf gesetzlicher Ebene ungeregelt bzw. lediglich Gegenstand von Verhandlungen zwischen Arbeitnehmervertretern und Management ist, so ist die Richtlinie doch bindend für alle Unternehmen, deren Beschäftigtenzahl den Bedingungen der Richtlinie entspricht, unabhängig vom Hauptsitz des Unternehmens. 
Stand der Forschung zu Europäischen Betriebsräten

\subsection{Allgemein}

Weil die ersten EBR schon vor Erlass der entsprechenden Richtlinie eingerichtet wurden und diese selber Ergebnis einer jahrzehntelangen Diskussion über Arbeitnehmermitwirkung auf transnationaler Ebene war, widmeten sich zumeist gewerkschaftsnahe Autoren auch schon früh dem Phänomen (Lecher und Platzer 1994; Schulten 1992; Jacquier 1997). Nach dem Erlass der Richtlinie wurden auch Juristen auf die Neuerung aufmerksam (Zügel 1995; Sandmann 1996; Rademacher 1996). Erste Serien von Fallstudien wurden ab 1997, also kurz nach Inkrafttreten der Richtlinie, durchgeführt (Deppe et al. 1997; Lamers 1998; Lecher et al. 1998; Lecher et al. 1999; Lecher et al. 2001; Müller et al. 2004). Schon früh setzte auch grundsätzliche Kritik an den Begrenzungen der Richtlinie ein: Streeck (1997) kam in seinem Aufsatz über die Entstehung der EBR-Richtlinie zum Schluss, bei den EBR handle es sich keinesfalls um Elemente einer "industrial citizenship" auf europäischer Ebene, sondern vielmehr um die Verlängerung nationalstaatlicher Mitwirkungsregime. Ähnlich skeptisch war Sadler (2000), der an Stelle einer Europäisierung im Gefolge der EBR eine Verschiebung von flächendeckenden Tarifverträgen hin zu unternehmensspezifischen, dezentralen Beziehungen der Sozialpartner befürchtete.

Einen methodischen Meilenstein für Fallstudien setzten Lecher et al. (1999, 60-72) mit der Typisierung von EBR nach ihrer Wirksamkeit in der Interaktion mit dem Management, den EBR-Mitgliedern untereinander, mit den Gewerkschaften sowie den örtlichen Arbeitnehmervertretungen. Die dort entwickelte Typologie wird später wiederholt aufgegriffen (Müller et al. 2004, 93-96; Kotthoff 2006). Die umfassendste Serie von Fallstudien, unter Berücksichtigung von insgesamt 41 EBR, unternahm ein international zusammengesetztes Forschungsteam im Auftrag der Europäischen Stiftung zur Verbesserung der Lebens- und Arbeitsbedingungen (Weiler 2004). ${ }^{5}$ Parallel dazu erschienen mehrere Sammelbände mit Beiträgen über EBR (Fitzgerald und Sterling 2004; Whittall et al. 2007; Hertwig et al. 2009). Neuere Fallstudien-Serien wie diejenige von Kotthoff (2006), Hauser-Ditz et al. (2010), Stöger (2011) oder jüngst Kerckhofs (2013), welcher Längsschnittanalysen und Vergleiche mit älteren Fallstudien vornimmt, werden von klaren methodischen Vorgaben geleitet. Stöger (2011) gelangt mit einer gegenüber den älteren Studien erweiterten Fragestellung zu einer neuen, aber noch immer an der Wirksamkeit orientierten Typologie von EBR. Die Monografie von Waddington (2011) schliesslich legt Ergebnisse einer Befragung von über 900 EBR-Mitgliedern aus verschiedenen EU-Ländern vor, welche zu einem skeptischen und zugleich vorsichtig optimistischen Schluss kommt in Bezug auf die Wirksamkeit der EBR-Richtlinie für eine Ausdehnung von Arbeitnehmerrechten. Neueste Forschung bezieht sich auf die

$5 \quad$ Kotthoff $(2006,29)$ bemerkt zu dieser Studie, die Fallstudien würden «nebeneinander gestellt, ohne den Versuch, sie zu komprimieren und Verallgemeinerungen aus ihnen zu ziehen». 
Mitwirkungs-Vereinbarungen in Europäischen Aktiengesellschaften (Eurofound 2011), auf die Bedeutung nationalkultureller Differenzen innerhalb von EBR (Klemm et al. 2011; Hürtgen 2011) sowie auf die namentlich in Gewerkschaftskreisen kritisch betrachtete Rolle von EBR beim Abschluss von transnationalen Unternehmensvereinbarungen (Eurofound 2009; Jagodzinski 2012; Müller et al. 2013; Rüb et al. 2013). Im Zusammenhang mit der hier vorgelegten Untersuchung interessieren zudem Beiträge, welche sich der Umsetzung bzw. Nicht-Umsetzung der Richtlinie in den transnationalen Unternehmen einzelner Länder widmen. Einige kommen zum Schluss, dass subjektive Faktoren auf Arbeitnehmerseite in den Stammländern dabei eine Rolle spielen - namentlich das Interesse bzw. Desinteresse der Stammland-Gewerkschaften bzw. Betriebsräte an der Einrichtung eines solchen länderübergreifenden Arbeitnehmergremiums (Bicknell 2007 für Deutschland; Costa und Araujo 2008 für Portugal). Für Deutschland konnte zudem nachgewiesen werden, dass die "compliance rate" in den zu mehr Transparenz verpflichteten börsenkotierten Unternehmen höher ist als in nicht kotierten, meist in Familienbesitz befindlichen Unternehmen. Denn um zu bestimmen, ob ein Unternehmen überhaupt unter die EBR-Richtlinie fällt, sind aussenstehende Akteure auf bestimmte Daten zum Beispiel über die Beschäftigtenzahlen angewiesen. Ausserdem nimmt bei deutschen Unternehmen die Wahrscheinlichkeit, dass ein EBR besteht, mit der Anzahl Beschäftigter zu (Lücking et al. 2008; Whittall et al. 2008).

\subsection{Schweiz und EBR}

Die vielfältige internationale Literatur über Europäische Betriebsräte hat sich der Tatsache, dass auf freiwilliger Basis in vielen Fällen die Arbeitnehmer der Schweizer Standorte einbezogen sind, bisher kaum gewidmet. Marginson et al. (1998, 21-22) analysieren zwar Art. 13-Vereinbarungen auf ihre Drittstaatenwirkung und den Einbezug der Schweiz, treffen aber keine Unterscheidung zwischen Unternehmen mit Standorten in der Schweiz und solchen ohne. Auf diese Erhebung stützt sich auch in jüngster Zeit noch Waddington $(2011,68)$. Lecher et al. (1998, 45-46) streifen den Bezug zum Nicht-EU-Land Schweiz kurz, ohne allerdings Erklärungen zu liefern für den partiellen Einbezug Schweizer Arbeitnehmer. Ein erster Versuch, die Relevanz von Europäischen Betriebsräten für die Schweiz systematisch zu erfassen, entstand im Rahmen eines interdisziplinären Sammelbandes über die Gewerkschaften in der Schweiz in den 1990er-Jahren (Ziltener 2000b, 265-274). Zuvor erschienen einzelne Beiträge aus der Sicht gewerkschaftlicher Praktiker (Baumann 1997, 62; Lüscher 1997). Zudem wurde innerhalb der Gewerkschaft Unia von Zeit zu Zeit versucht, sich einen Überblick über das Ausmass des Einbezugs der Schweiz in EBR zu verschaffen. Diese Listen blieben zumeist begrenzt auf Unternehmen im Organisationsbereich der Unia; zudem fielen auch Unternehmen namentlich im Dienstleistungssektor, zu denen kein gewerkschaftlicher Kontakt bestand, aus dem Blickfeld (Baumann 1997; Rennwald und Eger 2004, 111-113; Schweizerisches 
Sozialarchiv, Ar Unia EBR 07.02 und 07.03). Hinzu kommen einzelne Beiträge von juristischer Seite zur Bedeutung von EBR für die Schweiz (Handschin 1995; Müller 1999, 316; Wildhaber 2009; Geiser 2010).

Eine jüngst erschienene geschichtswissenschaftliche Dissertation über die Schweizer Gewerkschaften und Europa untersuchte erstmals Schweizer EBRVertretungen in drei transnationalen Unternehmen mit Hauptsitz in der Schweiz sowie die Rolle von gewerkschaftlichen Sachverständigen aus der Schweiz bei der Errichtung von zwei dieser EBR (Wyler 2012, 216-303). Wylers Untersuchung bleibt beschränkt auf Industrieunternehmen im Organisationsbereich der Unia und auf die Befragung von Protagonisten aus dieser Gewerkschaft. EBR von Schweizer Unternehmen wurden auch in anderen Fallstudien-Serien vereinzelt berücksichtigt (Lecher et al.1999; Lecher et al. 2001; Müller et al. 2004; Stöger 2011) sowie in einer vergleichenden Studie über die Rolle von Gewerkschaften und EBR bei grenzüberschreitenden Unternehmensfusionen (Erne 2008).

\section{Untersuchungsrahmen und Methoden}

\subsection{Einbezug der Schweizer Standorte in Europäischen Betriebsräten}

Sämtliche den Autoren vorliegenden Vereinbarungen über die Errichtung von EBR wurden im Hinblick auf die geografische Reichweite bzw. Aussagen über die Vertretung der Schweizer Standorte analysiert. Diese sind in der Regel auf der Datenbank des Europäischen Gewerkschaftsinstituts (EGI) zugänglich (European Trade Union Institute 2013), in einigen Fällen wurden sie den Autoren durch Insider zur Verfügung gestellt. Sämtliche Unternehmen bzw. Unternehmenseinheiten mit einem EBR (ebenfalls gemäss den Angaben des EGI) wurden zudem mittels Recherchen auf Firmenwebseiten und Telefonverzeichnissen daraufhin untersucht, ob sie in der Schweiz Arbeitnehmer beschäftigen. Gut zweihundert Unternehmen, deren Status bezüglich Einbezug der Schweizer Arbeitnehmer in den EBR unklar war, wurden angeschrieben; deren Rückmeldungen (bei einer Quote von ca. einem Drittel) flossen ebenfalls in die Untersuchung ein. Ergänzend wurden Expertengespräche mit zuständigen Vertretern von Arbeitnehmer- und Arbeitgeberverbänden sowie mit weiteren Insidern geführt. Die hier präsentierten Ergebnisse beruhen dementsprechend teils auf der Analyse der Vereinbarungen, teils auf Auskünften der Unternehmen und auf Angaben von Insidern.

Die Gesamtheit der Unternehmen mit EBR und Beschäftigten in der Schweiz beziffern wir auf 472 - bei 955 insgesamt bestehenden EBR. Dies bedeutet, dass sich bei der Hälfte aller Europäischen Betriebsräte zumindest potenziell die Frage stellt, ob Schweizer Arbeitnehmer in ihnen vertreten werden sollen.

Ziel dieser Untersuchung ist die Beantwortung der Frage, wovon eine Vertretung der Schweizer Standorte in den jeweiligen EBR abhängt. Wir gehen davon 
aus, dass vier Faktoren potenziell signifikant sind: die Branchenzugehörigkeit des Unternehmens (a), der Hauptsitz des Unternehmens (b), der Typ der dem EBR zugrunde liegenden Vereinbarung (c) sowie die Relevanz des Schweizer Standorts (d).

a) Angesichts der geringen Grösse der zu betrachtenden Grundgesamtheit und auch angesichts der teilweise grossen Vielfalt der Geschäftsfelder bei etlichen transnationalen Unternehmen drängte sich eine eher grobe Einteilung nach Branchen auf. Die Einteilung wurde ungeachtet der realen Tätigkeit des Unternehmens in der Schweiz vorgenommen; ein Industrieunternehmen also auch dann als solches klassifiziert, wenn es in der Schweiz über keinen Produktionsstandort, sondern lediglich über Service- oder Vertriebsstandorte verfügt. Die Unternehmen wurden in vier Kategorien eingeteilt: die Maschinen-/Elektro-/Metallindustrie (inkl. Elektronikunternehmen), die Chemie- und Pharmaindustrie, der Dienstleistungssektor (Finanzen, Transport und Logistik, Facility Management, Telekommunikation, Informatik, Touristik) sowie die Gruppe «Diverse Industrien", bestehend u.a. aus Bau- und Baustoff-, grafischer und Nahrungsmittelindustrie sowie der Restaurationsbranche.

b) Wir gehen davon aus, dass sich für Unternehmen mit Hauptsitz in der Schweiz angesichts politischer Sensibilitäten und auch angesichts der Bedeutung des Standorts die Frage einer Schweizer Vertretung im EBR grundsätzlich anders stellt als für andere Unternehmen. Es darf vermutet werden, dass, auch wenn die Vereinbarung arbeitgeberseitig in vielen Fällen von einer Niederlassung in der EU unterzeichnet wird, die in der Schweiz ansässige zentrale Leitung als Akteur in die Verhandlungen einbezogen war. Zudem interessieren hier allfällige Unterschiede zwischen europäischen (in der EU / EWR ansässige) und aussereuropäischen (namentlich US-amerikanischen, japanischen und australischen) Unternehmen.

c) Bis zum Inkrafttreten der Richtlinie im September 1996 geschlossene EBR-Vereinbarungen, die den Bestandesschutz von Art. 13 der Richtlinie geniessen, wurden auf Arbeitnehmerseite in der Regel von gewerkschaftlichen und / oder betrieblichen Vertretern unter starker Dominanz des Hauptsitzstaats und häufig auch eng begleitet von den jeweiligen europäischen Gewerkschaftsdachverbänden ausgehandelt. Dabei, so die Ausgangshypothese, war die Wahrscheinlichkeit gross, dass die EGB-Position eines Einbezugs der Schweizer Standorte (Waddington 2011; Wyler 2012) von gewerkschaftlicher Seite in die Verhandlungen eingebracht werden konnte. Bei später von sogenannten "Besonderen Verhandlungsgremien», denen Schweizer Vertreter höchstens auf freiwilliger Basis angehörten ${ }^{6}$, geschlossene Vereinbarungen ist diese Wahrscheinlichkeit geringer. Bei der entsprechenden Kodierung ausschlaggebend war nicht die aktuelle Vereinbarung, sondern vielmehr die erste im betreffenden Unternehmen oder in einem seiner Vorgängerunternehmen abgeschlossene, weil angenommen werden kann, dass der Status der Schweizer Ver-

6 Der Jurist Rademacher (1996, 134) vertrat sogar die Auffassung, der Einbezug von Angehörigen von Drittstaaten (also auch von Arbeitnehmervertretern aus der Schweiz) in Besondere Verhandlungsgremien sei höchstens mit Gaststatus, nicht aber mit vollem Stimmrecht zulässig. 
tretung sich in der Regel nicht mehr ändert. ${ }^{7}$ Nicht weiter beachtet werden in diesem Zusammenhang die EBR in Europäischen Aktiengesellschaften (SE-Betriebsräte, 27 Fälle) und die seltenen nach subsidiären Bestimmungen errichteten EBR in Unternehmen mit Schweizer Beschäftigten (7 Fälle) ${ }^{8}$ sowie diejenigen Fälle, bei denen der "EBR-Typ» nicht eruiert werden konnte (23 Fälle).

d) Schliesslich kann vermutet werden, dass jenseits der Frage, ob ein Schweizer Standort überhaupt über die vereinbarungsgemässe Mindestzahl an Beschäftigten (je nach Vereinbarung z. B. 50 oder 100) für eine EBR-Vertretung verfügt, die schiere Relevanz des Schweizer Standorts ebenfalls einen (positiven) Einfluss darauf hat, ob dessen Vertretung im EBR bei den Verhandlungen überhaupt ein Thema war und somit gegebenenfalls auch durchgesetzt werden konnte. Diese Ausgangshypothese wird durch die Tatsache genährt, dass gemäss dem Schweizer Mitwirkungsgesetz betriebliche Arbeitnehmervertretungen erst ab einer Mindestzahl von 50 Beschäftigten eingerichtet werden müssen, und auch dadurch, dass eine gewerkschaftliche Präsenz bei grösseren und namentlich bei Produktionsstandorten eher gegeben ist als bei reinen Verkaufs- oder Verwaltungsstandorten. Und sowohl die Existenz einer Arbeitnehmervertretung als auch die Präsenz von Arbeitnehmervereinigungen erhöhen die Wahrscheinlichkeit, dass bei den Verhandlungen über einen EBR oder im Falle einer nachträglichen Betriebsübernahme bei entsprechenden Nachverhandlungen die Vertretungsinteressen der Arbeitnehmer in der Schweiz auf europäischer Ebene geltend gemacht werden können. In Bezug auf diese Variable wurden alle Unternehmen diskretionär in die Kategorien «relevanter Standort» und «hinsichtlich seiner Beschäftigtenzahlen wenig relevanter Standort» eingeteilt. ${ }^{9}$

\subsection{Europäische Betriebsräte in schweizerischen Unternehmen}

Für die Beantwortung der Frage, welche transnationalen Unternehmen mit Hauptsitz in der Schweiz EBR-pflichtig sind und welche real über einen EBR verfügen, also die Frage nach der Umsetzungsquote oder "compliance rate” der Schweizer Unternehmen im Vergleich zu derjenigen aller EBR-pflichtigen Unternehmen, wurden verschiedene Quellen beigezogen: die Datenbank des EGI (European Trade Union Institute 2013), die Liste Top 2011 der Handelszeitung (Schweizerische Handels-

$7 \quad$ Es bestehen allerdings Anzeichen dafür, dass in einigen Fällen, die derzeit neu verhandelt werden bzw. bei denen Neuverhandlungen möglicherweise anstehen, die Schweizer Vertretung von Managementseite vermehrt in Frage gestellt wird. Dazu liegen aber derzeit noch keine gesicherten empirischen Erkenntnisse vor.

8 Bei diesen EBR muss ein Einbezug der Schweizer Standorte von vornherein ausgeschlossen werden, weil ja per definitionem keine Vereinbarung vorliegt, welche von den subsidiären Bestimmungen, gemäss denen die EBR-Richtlinie auf die Schweiz nicht anwendbar ist, abweichen könnte.

9 Blosse Verkaufsniederlassungen von Pharma- und Automobilkonzernen sowie Unternehmen mit nachweislich weniger als 100 Beschäftigten in der Schweiz wurden als "wenig relevant» kodiert. Unternehmen mit schweizerischen Produktions- und / oder Forschungs- oder Verkaufsstandorten (in jedem Fall bei Banken, Hotelketten, Detailhandelsunternehmen vermutet) oder grösseren Serviceeinheiten (in jedem Fall bei Büroelektronikherstellern vermutet) oder mit nachweislich mehr als 100 Beschäftigten wurden als «relevant» eingestuft. 
zeitung 2011) und der Swiss Market Index (Stand 2013). Berücksichtigt wurden Unternehmen mit Hauptsitz in der Schweiz mit mindestens 1000 Beschäftigten in EU / EWR-Staaten und nachweislicher Vertretung in mehr als zwei von der Richtlinie betroffenen Staaten und, sofern kein EBR besteht, einer relevanten Anzahl Beschäftigter in der Schweiz oder zumindest einer Kotierung an der Schweizer Börse: Reine Holdings oder "Briefkastenfirmen» gelten in diesem Zusammenhang nicht als Schweizer Unternehmen.

Als unabhängige Variable für die Erklärung der Frage, ob ein Unternehmen real über einen EBR verfügt, wurde im Anschluss an die entsprechende Studie für die "compliance rate" bei Unternehmen mit Hauptsitz in Deutschland (Lücking et al. 2008; Whittall et al. 2008) die Kotierung des Unternehmens an der Schweizer oder einer anderen Börse (Stand Ende 2013) herangezogen. Vermutet wird - in Anlehnung an die oben zitierte Studie - aufgrund der herrschenden Offenlegungspflichten ein positiver Zusammenhang zwischen Börsenkotierung und Einrichtung eines EBR. Die Beschäftigtenzahl konnte hingegen nicht als unabhängige Variable herangezogen werden, weil darüber - gerade bei nicht börsenkotierten Unternehmen - zu wenige gesicherte Angaben vorliegen.

\section{$4 \quad$ Resultate}

\subsection{Einbezug der Schweizer Standorte in Europäischen Betriebsräten}

Von den 472 in Frage kommenden EBR verfügen 159 über einen bis vier Schweizer Vertreter (vgl. Tabelle 1) - wobei in einigen Fällen diese Vertretung derzeit vakant ist. In zehn dieser Fälle sind Schweizer nur als Beobachter bzw. Gäste im EBR präsent, was beispielsweise bedeutet, dass das Management dem EBR über die Schweizer Ländergesellschaft keinerlei Informationen zukommen lässt. ${ }^{10}$ Hinzu kommen 24 EBR, in denen eine Vertretung der Schweizer Standorte durch andere, in der Regel Nachbarstaaten, vorgesehen ist, oder eine solche einzig aus dem Grund ausgeschlossen ist, weil die Mindestzahl von Beschäftigten, die für eine Vertretung vereinbart ist, nicht erreicht wird. In diesen Fällen liegt also kein Ausschluss aufgrund der Nichtmitgliedschaft der Schweiz in der EU bzw. dem EWR vor. In 248 Fällen ist überhaupt keine Vertretung der Schweiz vorgesehen, ${ }^{11}$ und in 41 Fällen konnte der Status nicht ermittelt werden. ${ }^{12}$

10 Quelle: Interview mit dem gewerkschaftlichen Sachverständigen beim EBR eines Schweizer Industrieunternehmens.

11 Dies bedeutet nicht, dass die Schweiz explizit ausgeschlossen würde; es genügt die explizite Beschränkung der geografischen Reichweite der jeweiligen EBR-Vereinbarung auf «EU» bzw. auf «EU und EWR».

12 «Unklarer Status» liegt bei Fällen vor, bei welchen die Vereinbarung nicht zugänglich oder bezüglich geografischer Reichweite unklar formuliert ist («alle europäischen Länder» oder «die Arbeitnehmer in Europa»), in der Vereinbarung die effektiv vertretenen Standortländer nicht genannt sind und wo das betreffende Unternehmen eine diesbezügliche schriftliche Anfrage nicht beantwortet hat. 
Tabelle $1 \quad$ Häufigkeit der verwendeten Variablen

\begin{tabular}{lrr}
\hline & Frequenz (in \%) & Fallzahl \\
\hline Reale Vertretung des Schweizer Standorts vorgesehen & & 431 \\
Ja & 36.89 & 159 \\
Nein & 63.11 & 272 \\
Branche & & 472 \\
Metall-/Elektro-/Maschinenindustrie & 37.08 & 175 \\
Chemie-/Pharmaindustrie & 18.43 & 87 \\
Dienstleistungen, inkl. Transport / Logistik / Facility Management / Tourismus & 22.67 & 107 \\
Diverse Industrien, inkl. Bau, Baustoffe, grafische und & 21.82 & 103 \\
Nahrungsmittelindustrie / Catering & & \\
Hauptsitz & & 472 \\
Schweiz & 10.59 & 50 \\
EU / EWR & 64.41 & 304 \\
Ausserhalb Europas & 25.00 & 118 \\
EBR nach Artikel 13 & & 415 \\
Ja & & 225 \\
Nein & 54.22 & 190 \\
Relevanter Schweizer Standort & 45.78 & 472 \\
Ja & & 303 \\
Nein & 64.19 & 169 \\
\hline
\end{tabular}

Insgesamt kann von derzeit knapp 230 EBR-Delegierten aus der Schweiz ausgegangen werden (Gabathuler und Ziltener 2013).

Die dichotome abhängige Variable «reale Vertretung des Schweizer Standorts» wurde in einem zweiten Schritt einer logistischen Regression unterzogen, wobei nur noch die Fälle mit geklärtem Status sowie zur Bestimmung des Einflusses der Variable «EBR-Typ» zusätzlich nur noch diejenigen mit einem EBR nach Art. 13 oder Art. 6 betrachtet wurden. Dadurch reduziert sich die Fallzahl von ursprünglich 472 auf 403 (vgl. Tabelle 2).

Gemäss diesem Modell erhöht die Zugehörigkeit zum Dienstleistungssektor die Wahrscheinlichkeit einer Vertretung im EBR signifikant; die Merkmale «Hauptsitz in der Schweiz», «EBR nach Art. 13» und "relevanter Schweizer Standort» sind sogar hoch signifikant (vgl. Tabelle 2).

Für dieses Modell wurden nun die durchschnittlichen marginalen Effekte (average marginal effects, AME) der signifikanten Variablen berechnet. ${ }^{13}$

Die durchschnittlichen marginalen Effekte zeigen, dass ein Hauptsitz in der Schweiz den stärksten Effekt (29.2\%) hat, also die Wahrscheinlichkeit am meisten steigert,

13 Zu den Problemen der gebräuchlicheren Odds Ratios siehe Mood (2010). 
Tabelle 2 Logistische Regression von realer Vertretung der Schweizer Standorte im EBR auf unabhängige Variablen

\begin{tabular}{lcc}
\hline & B-Koeffizient & Durchschnittliche marginale Effekte \\
\hline Zugehörigkeit zur Dienstleistungsbranche & 0.574 & 0.114 \\
& $(2.11)^{*}$ & $(2.16)^{*}$ \\
Hauptsitz in der Schweiz & 1.464 & 0.292 \\
& $(4.04)^{* *}$ & $(4.39)^{* *}$ \\
EBR nach Art. 13 errichtet & 0.704 & 0.140 \\
& $(3.07)^{* *}$ & $(3.07)^{* *}$ \\
Relevanter Schweizer Standort & 0.867 & 0.172 \\
& $(3.37)^{* *}$ & $(3.53)^{* *}$ \\
Konstante & -1.849 & \\
& $(7.12)^{* *}$ & \\
Pseudo R & 0.111 & \\
$\mathrm{~N}$ & 403 & \\
\hline
\end{tabular}

Anmerkungen: Nur signifikante Variablen angezeigt, z-Werte in Klammern; ${ }^{*} p \leq 0.05 ;{ }^{*} p \leq 0.01$.

dass eine Schweizer Vertretung im EBR existiert (vgl. Tabelle 2). Ebenso steigt die Wahrscheinlichkeit bei «relevanten Schweizer Standorten» (17.2\%) sowie bei EBR, die nach Art. 13 (14.0\%) errichtet wurden. Den geringsten positiven Effekt (11.4\%) hat die Zugehörigkeit eines Unternehmens zur Dienstleistungsbranche.

Die oben unter 3.1 präsentierten Ausgangshypothesen bezüglich Einfluss von Hauptsitz (b), «EBR-Typ» (c) und Relevanz des Schweizer Standorts (d) auf die Vertretungswahrscheinlichkeit werden also bestätigt; sämtliche vermuteten Einflussvariablen - Hauptsitz in der Schweiz, EBR nach Art. 13 (Einfluss des EGB) und Relevanz des Standorts - erweisen sich als hoch signifikant. Hinzu kommt ein schwacher signifikanter Einfluss der Branchenzugehörigkeit bei den Dienstleistungsunternehmen, der so nicht zu erwarten war. Bei dieser Korrelation handelt es sich gemäss unserer Interpretation nicht um einen kausalen Zusammenhang; zu möglichen Zusammenhängen zwischen Branchenzugehörigkeit und Vertretungswahrscheinlichkeit waren keine Ausgangshypothesen formuliert worden. Als nicht signifikant stellten sich in der logistischen Regression die Unterschiede zwischen den europäischen und den aussereuropäischen Unternehmen sowie die Unterschiede innerhalb der verschiedenen Branchen der verarbeitenden Industrie heraus.

\subsection{Europäische Betriebsräte in schweizerischen Unternehmen}

Gemäss unserer Erhebung sind 110 Unternehmen mit Hauptsitz in der Schweiz von der EBR-Richtlinie betroffen. Von diesen verfügen 50 über einen EBR oder - in seltenen Fällen - über ein richtlinienkonformes anderes «Verfahren zur Unterrich- 
tung und Anhörung der Arbeitnehmer». Die Schweiz ist damit hinter den USA und Japan der drittwichtigste Sitzstaat von Unternehmen mit EBR (European Trade Union Institute 2013), der sich ausserhalb des Geltungsbereichs der Richtlinie befindet, was die Bedeutung der Schweiz als Sitzstaat in Europa tätiger transnationaler Konzerne unterstreicht. Die Umsetzungsquote für Schweizer Unternehmen ist leicht höher als diejenige aller betroffenen Unternehmen - ob mit Hauptsitz innerhalb oder ausserhalb des EWR: 45.5\% für die schweizerischen Unternehmen im Vergleich zu 39.1\% für alle Unternehmen. Das EGI geht von insgesamt 955 Unternehmen mit einem EBR aus, bei 2445 mutmasslich betroffenen Unternehmen (European Trade Union Institute 2013). Von den 20 gewichtigsten an der Schweizer Börse kotierten Unternehmen, die den Swiss Market Index ausmachen, verfügen 14 über einen EBR (70\%). Unter sämtlichen börsenkotierten Unternehmen, die mutmasslich unter die Richtlinie fallen, haben 54.2\% einen EBR. Unter den nicht börsenkotierten Unternehmen mit Hauptsitz in der Schweiz, die von der Richtlinie mutmasslich betroffen sind, sind es lediglich 29.0\%. Die Ausgangsthese, wonach eine Börsenkotierung die Einrichtung eines EBR begünstigt, hat sich somit auch für die Schweiz bestätigt. Die gegenüber allen börsenkotierten Unternehmen besonders hohe "compliance rate" unter den SMI-Unternehmen kann auch als Indiz dafür gewertet werden, dass die Grösse eines Unternehmens die Einrichtung eines EBR positiv beeinflusst. Über diesen wie über weitere mögliche Bestimmungsgründe ${ }^{14}$ für die Einrichtung oder Nicht-Einrichtung eines EBR kann diese Untersuchung aber keine gesicherten Erkenntnisse liefern.

\section{$5 \quad$ Schluss und Ausblick}

Wie unsere Ergebnisse zeigen, wurde das quantitative Ausmass der Schweizer Beteiligung an Europäischen Betriebsräten, so es überhaupt die Aufmerksamkeit von wissenschaftlicher Forschung und verbandspolitischer Praxis erfahren hatte, bisher unterschätzt. Etwa die Hälfte aller Unternehmen mit einem EBR beschäftigt Arbeitnehmer in der Schweiz. Und in immerhin etwa einem Drittel dieser Unternehmen und in höherem Ausmass in solchen mit bedeutenden Schweizer Standorten sind die Schweizer Arbeitnehmer im jeweiligen EBR vertreten - auf rein freiwilliger Basis. Die signifikantesten Einflussfaktoren für eine solche Vertretung sind ein Hauptsitz in der Schweiz, die Relevanz der Schweizer Niederlassung hinsichtlich der Anzahl

14 Unter den von der Richtlinie mutmasslich erfassten nicht börsenkotierten Unternehmen ohne EBR befinden sich auch die genossenschaftlich organisierten Einzelhandelskonzerne Migros und Coop sowie die in Staatsbesitz befindliche Schweizerische Post. Bei diesen drei Unternehmen dürfte nicht die den Nichtkotierten unterstellte Verschwiegenheit (Lücking et al. 2008), sondern andere Gründe (namentlich die starke Dominanz des Stammlands mit entsprechendem Desinteresse der schweizerischen Gewerkschaften an der Einrichtung eines EBR) ausschlaggebend für die "non-compliance" sein. 
Beschäftigter sowie die Errichtung eines EBR vor 1996 aufgrund einer «freiwilligen Vereinbarung» nach Art. 13 der damaligen Richtlinie. Anders formuliert: Ein Unternehmen mit Schweizer EBR-Beteiligung hat dies typischerweise früh entschieden, verfügt über bedeutende Standorte (insbesondere Produktionsstätten) in der Schweiz, und der Einbezug der Schweizer Arbeitnehmer wurde von gewerkschaftlicher Seite aktiv betrieben. Auch für transnationale Unternehmen mit Hauptsitz in der Schweiz hat die EBR-Richtlinie, unabhängig von einer Schweizer Beteiligung im EBR, eine grosse Bedeutung: Die Schweiz ist einer der wichtigsten Sitzstaaten von Unternehmen mit EBR ausserhalb der EU. Das sind Evidenzen dafür, dass sich Schweizer Unternehmen dem Trend zur Europäisierung der betrieblichen Arbeitsbeziehungen nicht entziehen können - und oft auch nicht wollen. Zugleich aber hat das NichtEU-Mitglied Schweiz bislang auf staatlicher Ebene keine adäquate Antwort auf diesen Prozess finden können. Dies führt zu einer Verunsicherung der Akteure auf Arbeitgeber- wie auf Arbeitnehmerseite und verstärkt damit tendenziell auch den «voluntaristischen» Charakter der Regulierung der Arbeitsbeziehungen in der Schweiz.

Im Anschluss an die quantitative Analyse wird das an der Universität Zürich angesiedelte Nationalfonds-Projekt verschiedene Schweizer EBR-Vertretungen aus schweizerischen und ausländischen Unternehmen unterschiedlicher Branchen und unterschiedlicher Grösse genauer untersuchen. Dabei interessieren v. a. Fragen nach den Funktionen und dem konkreten Nutzen Europäischer Betriebsräte für das Management wie für die Arbeitnehmer und ihre Interessenverbände, nach der allfälligen speziellen Situation der Schweizer Vertreter als «Drittstaatenvertreter» und nach den für eine «gute EBR-Praxis» erforderlichen Bedingungen. Zu erwarten sind auch weitere Aufschlüsse über die konkreten Gründe einer Vertretung der Schweizer Standorte in den jeweiligen EBR sowie über die Hintergründe der Errichtung von EBR in Schweizer Unternehmen. Aus ersten mit Betroffenen sowie Gewerkschaftsvertretern geführten Interviews ist zum einen absehbar, dass die Variationsbreite der jeweils gemachten Erfahrungen und ihrer Bewertung durch die Betroffenen sehr gross ist. Die Qualifikationen für EBR reichen von «gutes Instrument für die Durchsetzung von Arbeitnehmerinteressen, falls man es zu nutzen weiss», ${ }^{15}$ bis zur völligen Nutzlosigkeit bzw. rein routinemässigen Veranstaltung. Die Verantwortung für eine als unbefriedigend empfundene EBR-Performance wird teils beim Management bzw. der ungenügenden inhaltlichen Ausgestaltung der jeweiligen Vereinbarung gesehen, teils aber auch bei den Kollegen im EBR selber, welche beispielsweise schlecht vorbereitet an die Treffen kämen oder zu sehr nur ihre nationalen Standortinteressen verträten. Schweizer Vertreter in EBR von ausländischen Unternehmen berichten, dass sie durch den direkten Kontakt mit der Konzernspitze teilweise über einen zeitlichen Informationsvorsprung vor dem eigenen lokalen Management verfügen. Diese Analyse wird auch den Spezifika der Arbeitsbeziehungen und der gewerk-

15 Quelle: Interview mit dem Schweizer EBR-Vorsitzenden eines Schweizer Unternehmens der Finanzbranche. 
schaftlichen Interessenvertretung - so die historisch gewachsene Vielfalt von im Selbstverständnis "gewerkschaftsfernen» Angestellten- und Berufsverbänden - in der Schweiz Rechnung tragen und so Erkenntnisse liefern können zur hierzulande sozialwissenschaftlich wenig erforschten und wie auf EU-Ebene, aber anders als in der Mehrheit der korporatistisch verfassten Staaten Kontinentaleuropas stark «voluntaristisch» geprägten Sozialpartnerschaft auf betrieblicher Ebene.

\section{$6 \quad$ Literaturverzeichnis}

Baumann, Hans. 1997. Schweiz und Europäische Betriebsräte - eine Zwischenbilanz. Die Volkswirtschaft 9: 60-63.

Bicknell, Helen. 2007. Ethno-, poly-, and Eurocentric European Works Councils. How does German involvement influence their identity? S. 111-131 in Towards a European Labour Identity: The Case of the European Works Council, hrsg. von Michael Whittall, Herman Knudsen und Fred Huijgen. Abingdon und New York: Routledge.

Böhringer, Peter. 2003. Mitbestimmung / Mitwirkung. S. 210 in Wörterbuch der Sozialpolitik, hrsg. von Erwin Carigiet, Ueli Mäder und Jean-Michel Bonvin. Zürich: Rotpunktverlag.

Costa, Hermes Augusto und Pedro Araujo. 2008. European companies without European Works Councils: Evidence from Portugal. European Journal of Industrial Relations 14(3): 309-325.

Deppe, Joachim, Reiner Hoffmann und Wieland Stützel (Hrsg.). 1997. Europäische Betriebsräte. Wege in ein soziales Europa. Frankfurt und New York: Campus.

Erne, Roland. 2008. European Unions. Labor's Quest for a Transnational Democracy. Ithaca und London: Cornell University Press.

Eurofound. 2009. European and International Framework Agreements: Practical Experiences and Strategic Approaches. Dublin / Luxembourg: European Foundation for the Improvement of Living and Working Conditions / Office for Official Publications of the European Communities.

Eurofound. 2011. Employee Involvement in Companies Under the European Company Statute. Dublin / Luxembourg: European Foundation for the Improvement of Living and Working Conditions / Publications Office of the European Union.

European Trade Union Institute. 2013. www.ewcdb.org. The database on european works council agreements. Brussels: ETUI, http://www.ewcdb.org (31.01.2013).

Fitzgerald, Ian und John Sterling (Hrsg.). 2004. European Works Councils: Pessimism of the Intellect, Optimism of the Will? Abingdon und New York: Routledge.

Gabathuler, Heinz und Patrick Ziltener. 2013. Die Bedeutung Europäischer Betriebsräte für die Schweiz wurde bisher unterschätzt. Die Volkswirtschaft - Das Magazin für Wirtschaftspolitik 7/8-2013: 51-54.

Geiser, Thomas. 2010. Stellenabbau bei Alstom. Europäischer Betriebsrat und Mitwirkungsrechte nach Schweizerischem Recht. Kurzstellungnahme. St. Gallen: Forschungsinstitut für Arbeit und Arbeitsrecht.

Handschin, Lukas. 1995. Die Schweiz als Sitzstaat eines Europäischen Betriebsrates. Schweizerische Zeitschrift für Wirtschaftsrecht 3/95: 132-137.

Hauser-Ditz, Axel, Markus Hertwig, Ludger Pries und Luitpold Rampeltshammer. 2010. Transnationale Mitbestimmung? Zur Praxis Europäischer Betriebsräte in der Automobilindustrie. Frankfurt: Campus.

Hertwig, Markus, Ludger Pries und Luitpold Rampeltshammer (Hrsg.). 2009. European Works Councils in complementary perspectives. Brussels: European Trade Union Institute.

Hürtgen, Stefanie. 2011. Europäische Interessenvertretung - eine Frage der Kultur? Industrielle Beziebungen 18(4): 315-335. 
Jacquier, Jean-Paul. 1997. Le comité d'entreprise européen. Une instance en devenir. Rueil-Malmaison: Editions Liaisons.

Jagodzinski, Romuald. 2012. European Works Councils and transnational company agreements - Balancing on the thin line between effective consultation and overstepping competences. S. 157-196 in Transnational Collective Bargaining at Company Level. A New Component of European Industrial Relations?, hrsg. von Isabelle Schömann, Romuald Jagodzinski, Guido Boni, Stefan Clauwaert, Vera Glassner und Teun Jaspers. Brussels: European Trade Union Institute.

Kerckhofs, Peter. 2013. European Works Council developments in times of crisis and in time of the implementation of the new EWC Directive. Beitrag an der ILERA Conference. Amsterdam, 20-22 Juni 2013.

Klemm, Matthias, Clemens Kraetsch und Jan Weynand. 2011. Solidarität in der europäischen betrieblichen Mitbestimmung als theoretische Herausforderung - ein kultursoziologischer Lösungsvorschlag. Industrielle Beziehungen 18(4): 292-314.

Kotthoff, Hermann. 2006. Lehrjahre des Europäischen Betriebsrats. Zehn Jahre transnationale Arbeitnehmervertretung. Berlin: edition sigma.

Lamers, Josee. 1998. The Added Value of European Works Councils. Haarlem: AWVN.

Lecher, Wolfgang und Hans-Wolfgang Platzer (Hrsg.). 1994. Europäische Union - Europäische Arbeitsbeziehungen? Nationale Voraussetzungen und internationaler Rahmen. Köln: Bund-Verlag.

Lecher, Wolfgang, Bernhard Nagel und Hans-Wolfgang Platzer. 1998. Die Konstituierung Europäischer Betriebsräte - Vom Informationsforum zum Akteur? Baden-Baden: Nomos.

Lecher, Wolfgang, Hans-Wolfgang Platzer, Stefan Rüb und Klaus-Peter Weiner. 1999. Europäische Betriebsräte - Perspektiven ihrer Entwicklung und Vernetzung. Baden-Baden: Nomos.

Lecher, Wolfgang, Hans-Wolfgang Platzer, Stefan Rüb und Klaus-Peter Weiner. 2001. Verhandelte Europäisierung. Die Einrichtung Europäischer Betriebsräte - Zwischen gesetzlichem Rahmen und sozialer Dynamik. Baden-Baden: Nomos.

Lücking, Stefan, Rainer Trinczek und Michael Whittall. 2008. Europäische Betriebsräte: Was lehrt der deutsche Fall für die Revision der EU-Richtlinie? WSI Mitteilungen 5/2008: 246-253.

Lüscher, Adrian. 1997. Europäische Betriebsräte in der Schweiz. Vergleichende Studie über die freiwilligen Vereinbarungen i.S. Art. 13 EU-Richtlinie 94/95/EG von Schweizer Unternehmen. Studie im Auftrag von GBI und SMUV. Zürich.

Marginson, Paul, Mark Gilman, Otto Jacobi und Hubert Krieger. 1998. Negotiating European Works Councils. An Analysis of Agreements Under Article 13. Luxembourg: Office for Official Publications of the European Communities.

Mood, Carina. 2010. Logistic regression: Why we cannot do what we think we can do, and what we can do about it. European Sociological Review 26(1): 67-82.

Müller, Roland A. 1999. Die Arbeitnehmervertretung. Bern: Stämpfli.

Müller, Torsten, Hans-Wolfgang Platzer und Stefan Rüb. 2004. Globale Arbeitsbeziehungen in globalen Konzernen? Zur Transnationalisierung betrieblicher und gewerkschaftlicher Politik. Eine vergleichende Fallstudie. Wiesbaden: VS Verlag für Sozialwissenschaften.

Müller, Torsten, Hans-Wolfgang Platzer und Stefan Rüb. 2013. Transnationale Unternehmensvereinbarungen und die Vereinbarungspolitik Europäischer Betriebsräte. Eine quantitative Analyse im Metallsektor. Düsseldorf: Hans-Böckler-Stiftung.

Oesch, Daniel. 2011. Swiss trade unions and industrial relations after 1990. A history of decline and renewal. S. 82-102 in Switzerland in Europe. Continuity and Change in the Swiss Political Economy, hrsg. von Christine Trampusch und André Mach. Abingdon und New York: Routledge.

Platzer, Hans-Wolfgang. 2002. Europäisierung und Transnationalisierung der Arbeitsbeziehungen in der EU. Internationale Politik und Gesellschaft 2002(2): 103-121. 
Platzer, Hans-Wolfgang und Torsten Müller. 2011. Global and European Trade Union Federations. A Handbook and Analysis of Transnational Trade Union Organizations and Policies. Bern: Peter Lang.

Rademacher, Ulf. 1996. Der Europäische Betriebsrat. Die Richtlinie 94/95/EG des Rates vom 22.9.1994 und ihr Umsetzung in nationales Recht. Baden-Baden: Nomos.

Rennwald, Jean-Claude und Alfred Eger. 2004. Eine Gewerkschaft, offen für Europa und die Welt. S. 107-117 in Keinen Schritt umsonst getan. Blicke auf die Gewerkschaft SMUV 1970-2000, hrsg. von Gewerkschaft Industrie, Gewerbe, Dienstleistungen SMUV. Baden: hier + jetzt.

Rüb, Stefan, Hans-Wolfgang Platzer und Torsten Müller. 2013. Transnational company bargaining and the Europeanization of industrial relations. Prospects of a negotiated order. Bern: Peter Lang.

Sadler, David. 2000. Organizing European labour: Governance, production, trade unions and the questions of scale. Transactions of the Institute of British Geographers, New Series 25(2): 135-152.

Sandmann, Bernd. 1996. Die Euro-Betriebsrats-Richtlinie 94/45/EG - Europäischer Betriebsrat und alternative Verfahren zur Unterrichtung und Anhörung der Arbeitnehmer in transnationalen Unternehmen. o. O.: C. F. Müller Verlag.

Schulten, Thorsten. 1992. Internationalismus von unten. Europäische Betriebsräte in Transnationalen Konzernen. Marburg: Verlag Arbeit \& Gesellschaft.

Schweizerische Handelszeitung. 2011. Top 2011. Die grössten Unternehmen in der Schweiz. Zürich: Schweizerische Handelszeitung.

Stöger, Harald. 2011. Abstieg oder Aufbruch? Europäische Betriebsräte zwischen Marginalisierung und transnationalem Einfluss. Wien und Berlin: LIT Verlag.

Streeck, Wolfgang. 1997. Industrial citizenship under regime competition: The case of the European Works Councils. Journal of European Public Policy 4(4): 643-664.

Thüsing, Gregor. 2011. Europäisches Arbeitsrecht. 2. neu bearbeitete Auflage. München: C. H. Beck.

Waddington, Jeremy. 2011. European Works Councils. A Transational Industrial Relations Institution in the Making. New York und Abingdon: Routledge.

Weiler, Anni. 2004. European Works Councils in practice. Dublin / Luxembourg: European Foundation for the Improvement of Living and Working Conditions / Office for Official Publications of the European Communities.

Whittall, Michael, Herman Knudsen und Fred Huijgen (Hrsg.). 2007. Towards a European Labour Identity: The Case of the European Works Council. Abingdon and New York: Routledge.

Whittall, Michael, Stefan Lücking und Rainer Trinczek. 2008. Understanding the European Works Council deficit in German multinationals. Transfer 14(3): 453-467.

Wildhaber, Isabelle. 2009. Entwicklungen im europäischen Arbeitsrecht: Zur Neufassung der Richtlinie über Europäische Betriebsräte 2009/38/EG und zu deren Auswirkungen für die schweizerische Praxis. ArbR, Mitteilungen des Instituts für Schweizerisches Arbeitsrecht 2009: 11-32.

Wyler, Rebekka. 2012. Schweizer Gewerkschaften und Europa 1960-2005. Münster: Westfälisches Dampfboot.

Ziltener, Patrick. 2000a. EC social policy: The defeat of the Delorist project. S. 152-183 in State-building in Europe. The Revitalization of Western European Integration, hrsg. von Volker Bornschier. Cambridge: Cambridge University Press.

Ziltener, Patrick. 2000b. Zwischen Interaktion und Integration: Das europäische Mehrebenensystem als Handlungsfeld der Schweizer Gewerkschaften. S. 219-289 in Gewerkschaften in der Schweiz. Herausforderungen und Optionen, hrsg. von Klaus Armingeon und Simon Geissbühler. Zürich: Seismo Verlag.

Zügel, Johannes. 1995. Mitwirkung der Arbeitnehmer nach der EU-Richtlinie über die Einsetzung eines Europäischen Betriebsrats. Frankfurt: Peter Lang. 